\title{
Frequency response and self-noise of the MET hydrophone
}

\author{
Dmitry L. Zaitsev, Svetlana Y. Avdyukhina, Maksim A. Ryzhkov, Iliya Evseev, Egor V. Egorov, and \\ Vadim M. Agafonov \\ The School of Electronics, Photonics and Molecular Physics, Moscow Institute of Physics and Technology, \\ Moscow 117303, Russia
}

Correspondence: Dmitry L. Zaitsev (zaitcev.dl@mipt.ru)

Received: 10 April 2018 - Revised: 9 July 2018 - Accepted: 11 July 2018 - Published: 20 July 2018

\begin{abstract}
An electrochemical hydrophone based on the principles of molecular electronic transfer (MET) has been described. The paper presents theoretical and experimental results for the sensitivity and the level of selfnoise determination for the MET hydrophone (METH) in the frequency range of $0.02-200 \mathrm{~Hz}$, which determines the fields of acceptance of the devices being developed. An experimental model has been developed by using a force-balancing feedback. Different methods and techniques for its calibration have been developed. The experimental device with $0.75 \mathrm{mV} \mathrm{Pa}^{-1}$ sensitivity flat in the frequency band $0.02-200 \mathrm{~Hz}$ has been presented. It has been demonstrated that in the ultra-low-frequency range METH noise could be much lower than the standard Wenz noise model. Easy to produce, cheap and suitable for mass production, the MET hydrophone could be in demand in marine and land acoustic research.
\end{abstract}

\section{Introduction}

At present, there is a great variety of pressure sensors and the fields of their application in the world (Sherman and Butler, 2007; Gautschi, 2002). Sensors are the primary source of information about the processes taking place in the world ocean (Asolkar et al., 2017; Bradley and Nichols, 2015). Hydrophones provide navigation and location of vessels (Lasky et al., 2004), fishing technologies for the fishing industry (Mismund, 1997), scientific research of the underwater biosphere (Slotte et al., 2004), and work of underwater acoustic communications (Kopp et al., 2000). Another field of application is the seismic exploration of minerals; hydrophones are used as part of towed braids and stationary bottom stations. A significant difference in this direction of applications is the requirement of high sensitivity in the ultra-lowfrequency range of $0.1-100 \mathrm{~Hz}$, as well as the possibility of registering extremely weak signals of pressure variation. Previous-generation technologies, such as piezoelectric (de Medeiros et al., 2015), electrostrictive, magnetostrictive and electrostatic sensors, could not be so effective in the range of ultra-low-frequency measurements. In these circumstances, there is a problem of developing pressure sensors based on the new technological principles that can meet the growing demands of engineering and scientific tasks. Among the popular scientific and technical developments, there are several directions. For example, an electromagnetic hydrophone consisting of a conventional wire and a magnet can be used to measure acoustic pressure (Grasland-Mongrain et al., 2012), and another physical principle is used in photonic hydrophones with a relatively high sensitivity. They are created based on the interaction of two polarized lasers from an optical fibre (Liu et al., 2016). Another type of acoustic sensor can be based on the effect of optical reflection on the fibre end (Shen et al., 2011), Bragg fibre gratings (Tan et al., 2011) or Fabry-Perot interferometers (Kim et al., 2014; Ma et al., 2016). The fibre optic hydrophone has been shown to have a good linear response with a high sensitivity and a high-pressure resolution. Another direction is the development of pressure sensors based on the MEMS technology (Xu et al., 2016). The sensitivity and the bandwidth of such devices can be quite high in the frequency range of $20 \mathrm{~Hz}-$ $1 \mathrm{kHz}$, which is sufficient for underwater acoustic detection at low frequencies. At the same time, many leading companies produce hydrophones based on the traditional piezoelectric effect (available at: https://www.bksv.com/en/products/ 
(last access: 7 July 2018), 2018) and Teledyne Marine TC4032 (Teledyne Marine publication, 2018).

A comparatively new and successfully proven technology based on the molecular electronic transfer (MET) (Hurd and Lane, 1957) in the field of seismology and geophysical research (Deng et al., 2016), navigation and motion control (Zaitsev et al., 2016), earthquake-proof construction (Antonovskaya et al., 2017), and offshore exploration (Agafonov et al., 2017) can be used to develop pressure sensors other than traditional piezoceramic, micromechanical and fibre-optic technologies. Distinctive features of sensors based on the MET are of extremely high sensitivity in the field of low frequencies and low level of self-noise.

The results of the work are presented in three main parts. In the first part, the principles of the device for developing an acoustic pressure sensor based on the molecular-electronic transfer technology are discussed. In the second part, theoretical operational principles are analysed and a theoretical model of noise in the working frequency band is formed; the third part is devoted to the experimental studies of the possibility of creating a MET hydrophone, principles of calibration, sensitivity and self-noise of prototypes. The model of its self-noise is built based on the previous knowledge of the physical processes responsible for noise in the MET sensor systems (Kozlov and Safonov, 2003). Including such physical mechanisms as convective noise (Safonov, 2003), noise caused by the impedance of the transforming element (Shabalina, 2007), hydrodynamic noise and noise due to the geometry of the electronic node (Zaitsev et al., 2015), and noise due to cross axis sensitivity (Zaitsev et al., 2018a) has been considered.

\section{What is the MET hydrophone?}

The fundamentals of the technology and the physical principles of the MET sensor devices are discussed in the teaching materials (Lidorenko et al., 1984), while at the same time there are many current reviews on this topic and articles in periodicals (Huang et al., 2013) and patents (Abramovich and Kharlamov, 2003). In the literature, one can find information on the current state of developments based on the MET, development vectors and key achievements of scientific groups. As for the operational principles of the devices based on the MET, they are analysed in detail in Agafonov et al. (2013).

The main element of the MET is the transforming electrode cell, placed in a concentrated electrolyte solution (Fig. 1). The composition of the solution is selected in such a way as to enable the reversible electrochemical oxidationreduction reaction to proceed on the electrodes. For these purposes, a so-called iodine iodide electrolyte is most often used. An example of such an electrolyte is a concentrated $\left(\sim 4 \mathrm{ML}^{-1}\right) \mathrm{KI}$ aqueous solution with the addition of a relatively small amount of molecular iodine $\mathrm{I}_{2}$. In the solution, almost complete dissociation of KI into negatively charged ions of I and positive ions of $\mathrm{K}^{+}$occurs, and molecular iodine reacts with $\mathrm{I}^{-}$ions to form a triiodide:

$\mathrm{I}_{2}+\mathrm{I}^{-} \rightarrow \mathrm{I}_{3}^{-}$.

In this case, the migration conductivity is practically absent, and the current flow through the electrodes is completely determined by the diffusion component. Thus, the process of electron transfer between the anode and the cathode is carried out by diffusion of ionized molecules (ions) in the electrolyte solution, and only the electronic exchange occurs on the electrodes themselves: the electron is taken at the cathode and is transferred to the external circuit at the anode. If a small potential difference $(<0.9 \mathrm{~V})$ is applied to the electrodes placed in the solution, reversible electrochemical reactions with electron transfer through the interface metalelectrolyte solution occur on the electrodes.

The reduction of the triiodide on the cathode:

$\mathrm{I}_{3}^{-}+2 e \rightarrow 3 \mathrm{I}^{-}$.

The oxidation of iodine at the anode:

$3 \mathrm{I}^{-}-2 e \rightarrow \mathrm{I}_{3}^{-}$.

At that, the potassium ions play the role of a background electrolyte and do not participate in the reactions. In this case, the distribution of the concentration of the active component $\mathrm{I}_{3}^{-}$is established in the system (Fig. 2a). This gradient line shows the $\mathrm{I}_{3}^{-}$spatial distribution along the axis of sensor sensitivity in a very simple single-dimensional model. Transport of triiodide ions without electric migration is described by a convective diffusion equation. The hydrodynamic velocity is determined by a Navier-Stokes equation for a noncompressible liquid (Larcam, 1965). In turn, in a stationary electrolyte, the speed of delivery is determined by the rate of diffusion of $\mathrm{I}_{3}^{-}$. In the presence of hydrodynamic flows, convective transport is added to the diffusion flow, which leads, depending on the direction of the flow in the fluid, to an increase or decrease in the current in the system. In other words (Fig. 2b), the fluid flow resulting from the mechanical motion distorts the stationary "motionless" concentration distribution of the charge carriers (ions of $\mathrm{I}_{3}^{-}$in the liquid) in the interelectrode space, which leads to a strong change in the concentration gradient near the electrode surface, and the electric current through the electrode, in turn, depends on the concentration gradient near the electrode surface as follows:

$\mathrm{I}=-D q \oint_{S}(\nabla c \mathrm{~d} S)$,

where $D$ denotes the $\mathrm{I}_{3}^{-}$diffusion coefficient, $c$ denotes the $\mathrm{I}_{3}^{-}$concentration, $q$ denotes the carrier charge, and $S$ denotes the electrode surface. Variations of the electric current due to the arising hydrodynamic flows are the output signal of 


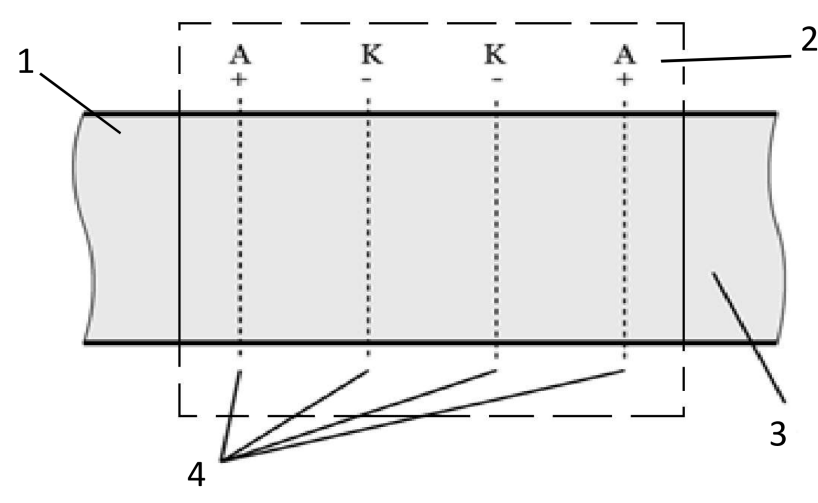

Figure 1. Transforming electrode cell (MET). 1 - channel walls; 2 - electrical package; 3 - electrolyte; 4 - mesh electrodes (external anodes $\mathrm{A}$, internal cathodes $\mathrm{K}$ ).

(a)

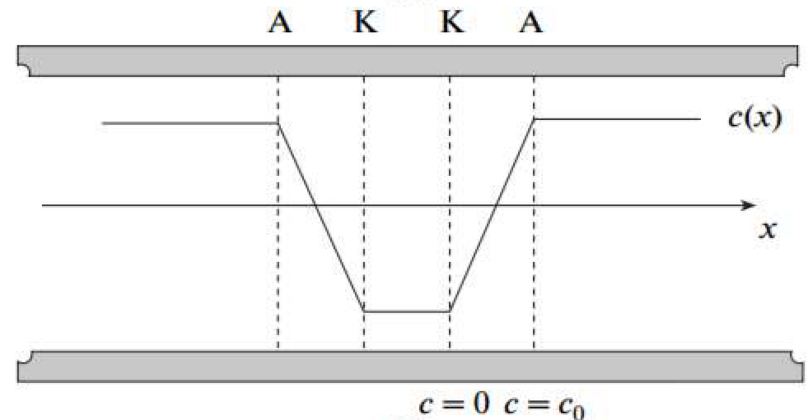

(b)

A $\quad K^{(b)} \mathrm{K} \quad \mathrm{A}$

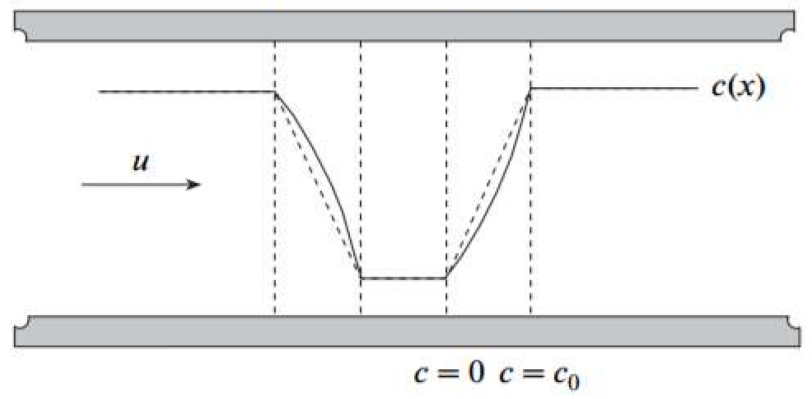

Figure 2. Distribution of the electrolyte concentration in the electrode assembly with mesh permeable electrodes. $c(x)$ is the steadystate concentration of triiodide ions; (a) concentration distribution without mechanical motion; (b) shows how the distribution of concentration $c(x)$ varies under the influence of the oncoming flow of liquid; $u$ - fluid flow rate. $\mathrm{A}$ - anodes, $\mathrm{K}$ - cathodes.

the MET sensor. There are a lot of scientific works on this theme. They take into consideration the dimensions of the system, the form of the electrodes, the size of the system, the different boundary conditions, and so on (Egorov et al., 2007).

The design and basic operational principles of a closedloop molecular electronic hydrophone (METH) are shown

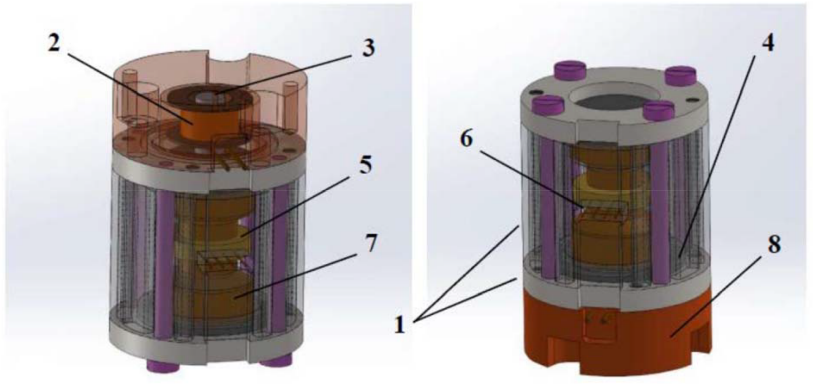

Figure 3. Constructional parts of the MET hydrophone. 1 - external body; 2 - coil; 3 - magnet; 4 - membranes; 5 - electrical package; 6 - electrical terminals of anodes and cathodes; 7 - electrolyte; 8 cover with air bubble under it.

in Fig. 3. It is designed on the basis of the construction of the MET closed-loop seismic accelerometers (Egorov et al., 2017) and the basic principles were approved in Zaitsev et al. (2018b). The sensing element of the MET sensor consists of two pairs of electrodes (cathode-anode) forming a so-called electrical package, as shown in Fig. 1. The electrical package is placed into a channel, bounded by rubber membranes (4) and filled with an electrolyte (7); 1 denotes the external sensor housing. A neodymium magnet (3) is glued to one of the rubber membranes, which can freely move inside the coil (2). The coil (2), in its turn, is rigidly adhered to the upper cover (8) (the insulating part of the volume of air from the external medium), so that the magnet can move inside it under the action of the Lawrence force. Such a simple scheme, on the one hand, makes it possible to introduce closed-loop feedback into the mechanical system, and on the other hand, it allows the hydrophone to self-calibrate.

The METH signal is the output current from the electrical terminals of the cathodes. As the two membranes of the sensor could be under different pressures, tiny pressure variations on the open membrane of the hydrophone can transform into a flow of working fluid. Further, the signal current passes through the correction, amplification and filtering circuits and the output of the electronic board is $\mathrm{I}_{\text {out }}$. The METH output signal is obtained by adding the signal current passing through the straight line $W_{\mathrm{f}}$, as well as the current passing through the feedback loop $W_{\mathrm{fb}}$ and fed to the coil (2). The feedback current applied to the coil causes the appearance of an electromagnetic field in the solenoid that interacts with the magnet (3), and by a rigid connection with the membrane creates a counterforce that balances the pressure drop caused by external acoustic influences on the sensor body. At the end, the output signal is processed by an analogue filter $W_{\text {filter }}$, providing a given operating frequency band. The mathematical model of the transfer characteristic of a sensitive element and associated electronic circuits can be written 


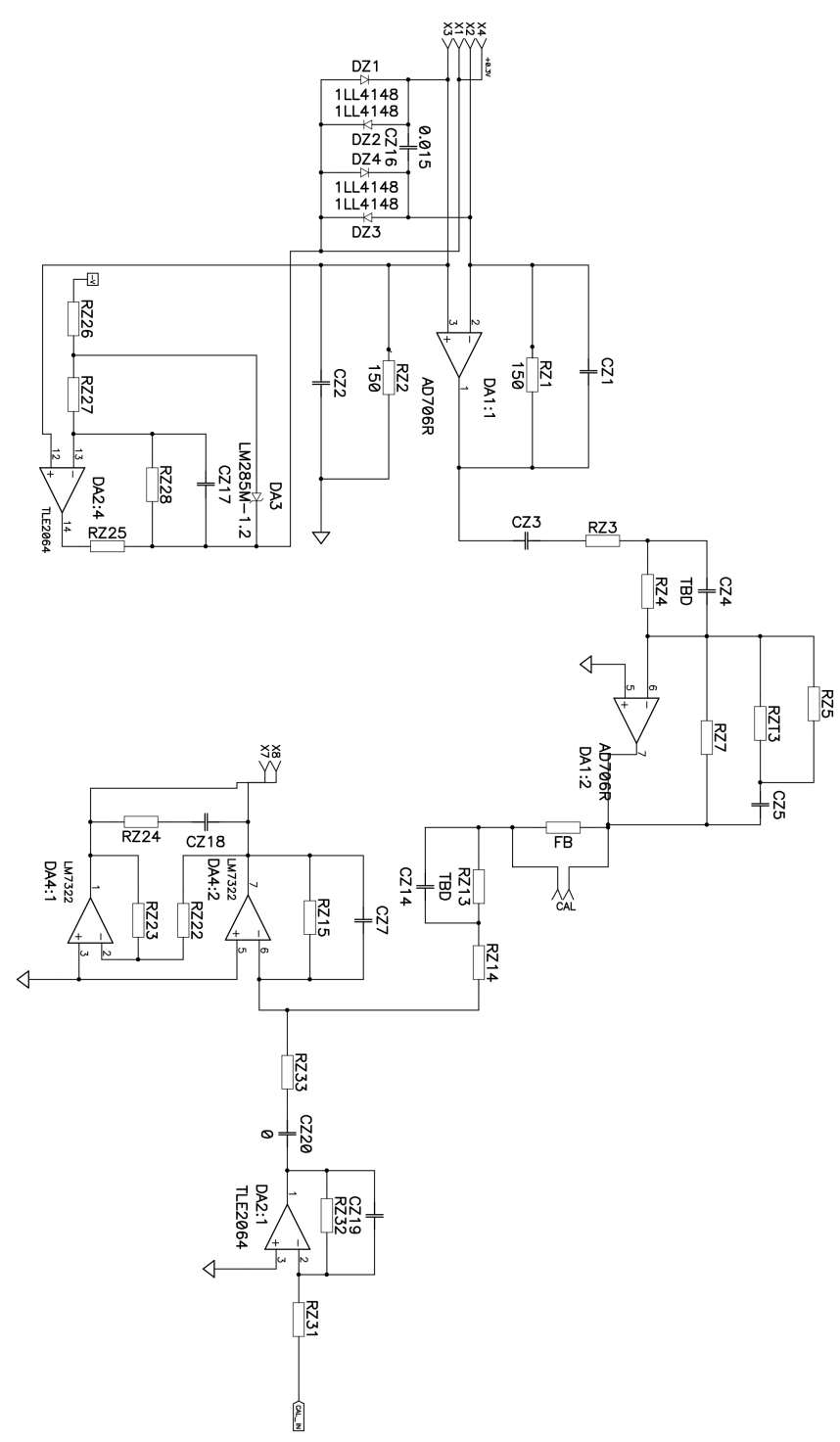

Figure 4. The MET hydrophone electronic circuit.

as follows:

$$
W=\frac{W_{\mathrm{s}}, W_{\mathrm{f}}}{1-W_{\mathrm{s}} \cdot W_{\mathrm{f}} W_{\mathrm{fb}}} \cdot W_{\text {filter }},
$$

where $W_{\mathrm{s}}=\mathrm{I}_{\mathrm{out}} / P$ means the METH sensor transfer function and denotes the measured pressure. $W_{\mathrm{f}}$ and $W_{\mathrm{fb}}$ are feedforward and feedback electronic transfer functions.

\section{Modelling section}

\subsection{Mathematical model of the hydrophone}

As was mentioned above, the hydrophone has been constructed based on the design of the MET closed-loop seismic accelerometer. However, these devices have significant differences. The main difference is the membrane function.
One of the two membranes separates the electrolyte solution from the air chamber, where the coil (2) and magnet (3) are located. Thereby the ambient pressure can be measured, because the pressure in the air chamber changes when the membrane deforms. Consider a simplified ideal gas model for gas enclosed between the membrane and the upper cover -8 . The parameter $c$ of the air chamber can be found from Boyle's law:

$c=p_{0} V_{0}=p\left(V_{0}-s_{\mathrm{m}} x\right)=\mathrm{const}$,

where $s_{\mathrm{m}}$ denotes the membrane surface and $x$ denotes the membrane displacement. Consider the system behaviour under the influence of small-amplitude harmonic oscillation of pressure. The volume of the electrolyte solution flowing through the MET's channels per unit of time can be found from Poiseulle's law:

$$
\begin{aligned}
Q=s_{M} \dot{x} & =\frac{p_{1}-p_{2}-\frac{M \ddot{x}}{s_{3}}}{R_{\mathrm{h}}} \\
& =\frac{p_{0}\left(1+m e^{i \omega t}\right)-\frac{p_{0} V_{0}}{V_{0}-s_{M} x}-\frac{M \ddot{x}}{s_{\ni}}}{R_{\mathrm{h}}},
\end{aligned}
$$

where the addendum $\frac{M \ddot{x}}{S_{\mathrm{ef}}}$ is the average force per unit square applied perpendicularly to a cross section of the liquid, $Q$ denotes the volume flow through the MET's channel per unit of time, $x$ denotes the membrane's displacement, $p_{1}$ denotes the external pressure, $p_{2}$ denotes the pressure in the air chamber, $s_{\mathrm{m}} \sim 100 \mathrm{~mm}^{2}$ is the membrane's surface, $s_{\mathrm{ef}} \sim 70 \mathrm{~mm}^{2}$ is the effective (average) cross-sectional square of a vessel, $p_{0}$ is the external pressure without impact, $m$ is the coefficient of pressure modulation, $V_{0} \sim 1 \mathrm{~cm}^{3}$ is the volume of the air chamber without impact, $M$ is the mass of the electrolyte solution, $R_{\mathrm{h}} \sim 3 \times 10^{9} \frac{\mathrm{N} \cdot S}{\mathrm{~m}^{5}}$ is the hydrodynamic impedance of the transducer, and $\omega$ is the angular frequency of the external pressure. If $s_{\mathrm{m}} x$ is small in comparison with $V_{0}$, (2) will have a form of

$\frac{p_{0} V_{0}}{V_{0}-s_{\mathrm{m}} x} \approx \frac{p_{0} V_{0}}{V_{0}}\left(1+\frac{s_{\mathrm{m}}}{V_{0}} x\right)=p_{0}+\frac{p_{0} s_{\mathrm{m}}}{V_{0}} x$.

Substitution of Eq. (4) into Eq. (3) gives the equation of membrane oscillations:

$\ddot{x}+\frac{s_{\mathrm{ef}} s_{\mathrm{m}} R_{\mathrm{h}}}{M} \dot{x}+\frac{p_{0} s_{\mathrm{ef}} s_{m}}{V_{0} M} x=\frac{p_{0} s_{\mathrm{ef}} m e^{i \omega t}}{M}$.

Self-system oscillations will be ignored; therefore, the system behaviour will be determined by the external impact. The real part of the solution of Eq. (5) is presented as

$$
x=\frac{-\left(\omega^{2}-\frac{p_{0} s_{\mathrm{ef}} s_{\mathrm{m}}}{V_{0} M}\right) \cos \omega t+\left(\omega \frac{s_{\mathrm{ef}} s_{\mathrm{m}} R_{\mathrm{h}}}{M}\right) \sin \omega t}{\left(\omega^{2}-\frac{p_{0} s_{\mathrm{ef}} s_{\mathrm{m}}}{V_{0} M}\right)^{2}+\left(\omega \frac{s_{\mathrm{ef}} s_{\mathrm{m}} R_{\mathrm{h}}}{M}\right)^{2}} \frac{p_{0} s_{\mathrm{ef}} m}{M} .
$$




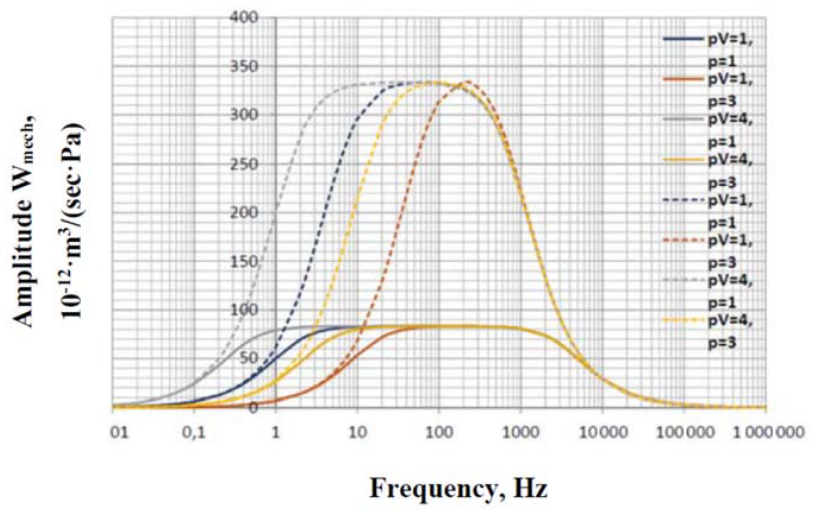

Figure 5. Spectrum of the mechanical transfer function. $R_{h}=$ $12 \times 10^{9} \frac{\mathrm{N} \mathrm{s}}{\mathrm{m}^{5}}$; the size of the channel is $3 \times 3 \mathrm{~mm}$ (solid curves). $R_{h}=3 \times 10^{9} \frac{\mathrm{N} \mathrm{s}}{\mathrm{m}^{5}}$; the size of the channel is $6 \times 6 \mathrm{~mm}$ (dotted curves). $R_{h} \sim \frac{1}{S_{\mathrm{el}}}$, where $S_{\mathrm{el}}$ is the electrode net surface, $S_{\mathrm{ch}}$ is the electrode channel surface, and $S_{\mathrm{el}} \sim S_{\mathrm{ch}}$ for the equal types of net.

Then, using (3) and (6),

$$
\begin{aligned}
& Q=\frac{\left(\omega^{2}-\frac{p_{0} s_{\mathrm{ef}} s_{\mathrm{m}}}{V_{0} M}\right) \sin \omega t+\left(\omega \frac{S_{\mathrm{ef}} s_{\mathrm{m}} R_{\mathrm{h}}}{M}\right) \cos \omega t}{\left(\omega^{2}-\frac{p_{0} S_{\mathrm{e}} s_{\mathrm{m}}}{V_{0} M}\right)^{2}+\left(\omega \frac{S_{\mathrm{ef}} s_{\mathrm{m}} R_{\mathrm{h}}}{M}\right)^{2}} \\
& \frac{\omega p_{0} S_{\mathrm{ef}} S_{\mathrm{m}} m}{M} .
\end{aligned}
$$

Therefore, the mechanical transfer function is presented by the following formula:

$$
\begin{aligned}
& W_{\text {mech }}=\left|\frac{Q}{p}\right|=\left|\frac{Q}{m p_{0}}\right|=\frac{1}{\sqrt{\left(\omega^{2}-\frac{p_{0} s_{3 \phi} s_{M}}{V_{0} M}\right)^{2}+\left(\omega \frac{s_{3 \phi} s_{M} R_{\mathrm{h}}}{M}\right)^{2}}} \\
& \frac{\omega S_{\ni \phi} S_{M}}{M} .
\end{aligned}
$$

Figure 5 shows the spectrum of the mechanical transfer function for two sizes of the square channel (solid lines $3 \times 3 \mathrm{~mm}$, dotted lines $-6 \times 6 \mathrm{~mm}$ ) and different external pressures and parameters of the air chamber (blue curves $c=1 \mathrm{~atm} \mathrm{c^{3 }}, p=1 \mathrm{~atm}$; orange curves $-c=1 \mathrm{~atm} \mathrm{c^{3 }}$, $p=3 \mathrm{~atm}$; grey curves $-c=4 \mathrm{~atm} \mathrm{~cm}^{3}, p=1 \mathrm{~atm}$; yellow curves $\left.-c=\operatorname{atm~cm}{ }^{3}, p=3 \mathrm{~atm}\right)$.

Note that the overall transfer function $W_{\mathrm{s}}$ consists of a unique part, $W_{\text {mech }}$ for the METH, which models for different pressures and volumes of the air chamber (Fig. 5), and $W_{\text {el-ch }}$ is the electrochemical part of the transfer function determining conversion of the electrolyte flow to the cathode current (Kozlov and Terent'ev, 2003).

\subsection{METH self-noise model}

Since the MET technology has been studied rather well, let us try to model the METH self-noise using knowledge of the existing noise mechanism in the MET. According to Kozlov and Sakharov (1994), at low frequencies the spectral density of hydrodynamic noise given in units of equal pressure is frequency independent and presented by the following formula:

$<p^{2}>_{\text {hydro }}=2 k T R_{h}$.

This noise is generated from hydrodynamic fluctuation of the electrolyte through the electrochemical MET cell. From Safonov (2003) and Zaitsev et al. (2015) we know that there is another noise type connected to arising of the close vortex in the channel. This noise is called geometrical and is given by the formula

$<p^{2}>_{\text {geom }}=\frac{4 k T}{R_{\mathrm{h}}\left|W_{\text {mech }}\right|^{2}} \alpha$,

where $\alpha$ is the dimensionless empirical coefficient, which characterizes scatter of the transfer function for different micro-channels of the electrochemical MET cell. Numerical value $\alpha=0.31$ is calculated in Zaitsev (2015). Moreover, there is noise described in Safonov (2003) that is generated from convection.

$$
\begin{aligned}
& <p^{2}>_{\mathrm{conv}}=\frac{K\left(\omega, s_{\mathrm{el}}\right)}{W_{\mathrm{mech}}^{2} W_{\mathrm{el}-\mathrm{ch}}^{2} R_{\mathrm{fb}}^{2}}, \\
& W_{\mathrm{el}-\mathrm{ch}}=\frac{I}{Q}=\frac{2 n e}{\sqrt{1+\frac{\omega^{2}}{\omega_{\mathrm{d}}^{2}}}},
\end{aligned}
$$

where $W_{\text {el-ch }}$ is the electrochemical transfer function of MET, $R_{\mathrm{fb}}$ is the feedback resistance of the first cascade of electronics, $e$ is the electron's charge, $n$ is the active electrolyte component concentration, $\omega_{\mathrm{d}}=\frac{D}{d^{2}}, D$ is the diffusion coefficient, and $d$ is the gap between the electrodes. The coefficient $K\left(\omega, s_{\mathrm{el}}\right)$ characterizes convection. The numerical value of $K\left(\omega, s_{\mathrm{el}}\right)$ for $s_{\mathrm{el}}=15.7 \mathrm{~mm}^{2}$ and the electrodes' channel $6 \times 6 \mathrm{~mm}$ has been calculated in Safonov (2003) and Zaitsev (2015).

$K\left(f, s_{\mathrm{el}}\right)=\left\{\begin{array}{l}1.68 \times 10^{-11} f \leq 1 \mathrm{~Hz} \\ 1.68 \times 10^{-11} f^{-1.1} f>1 \mathrm{~Hz}\end{array}\right.$

Modelling results of the METH self-noise and prediction of pressure variation influence and the volume of the air chamber are presented in Fig. 6 .

\section{Experimental section}

\subsection{The experimental set-up}

The first question of the research: how to calibrate the MET hydrophone at a very low frequency $(0.01-100 \mathrm{~Hz})$. To solve this problem, an experimental calibration set has been designed (Fig. 7).

The experimental set consists of a stiff container filled with water (1) with the MET hydrophones and reference piezoelectric hydrophone on its floor. The container (1) has an 


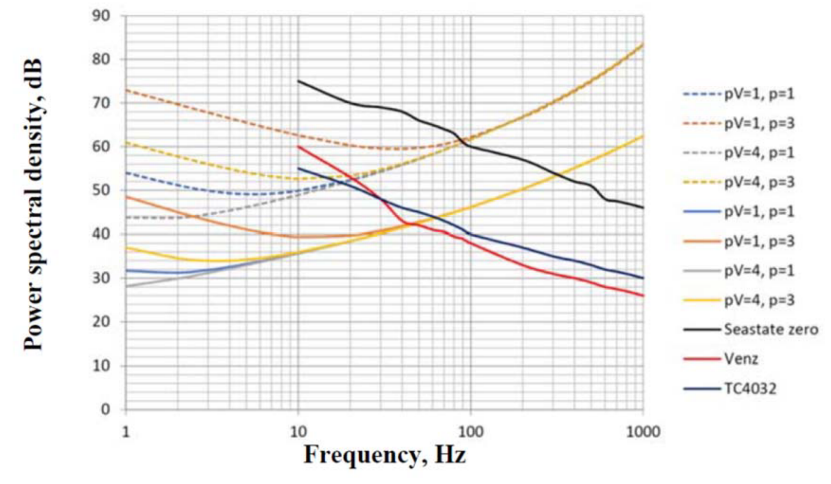

Figure 6. METH noise modelling for different $\mathrm{pV}$ and $p$ parameters of the METH air chamber in comparison with the Wenz model (Wenz, 1962) and some modern piezo hydrophones (TC-4032, available at: http://www.m-b-t.com, last access: 12 July 2018). Hz $-x$ axis, $\mathrm{dB}($ re $1 \mu \mathrm{Pa} / \sqrt{\mathrm{Hz}})-y$ axis.

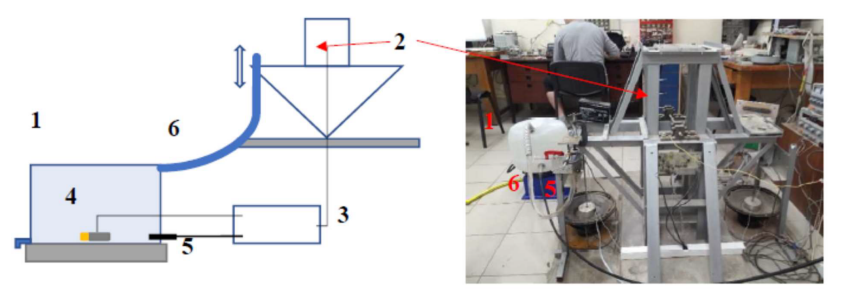

Figure 7. Principal design and view of the MET hydrophone calibration set. 1 - container with water and hydrophones; 2 - vertical offset generator; 3 - DA converter/digitizer; 4 - the MET hydrophone; 5 - piezoelectric hydrophone BC-311; 6 - open-end pipe with water.

output pipe (6) with an open end. The pipe is raised on the level of height, so that the water column makes additional pressure inside the container (1). A tilting calibration platform (2) was used to change the pressure. Its main part is a platform $300 \mathrm{~mm}$ high, $600 \mathrm{~mm}$ long and $400 \mathrm{~mm}$ wide. This heavy construction (over $60 \mathrm{~kg}$ ) is suspended on a rigid torsion bar. Attention is paid to providing both static and dynamic stiffness in the operating frequency range. The calibration process is controlled by a computer with the appropriate software. Sinusoidal oscillations are set by a 12-bit L-card digital-to-analogue converter, the signal from which, passed through a smoothing filter, is fed through a power amplifier to a pair of powerful $(250 \mathrm{~W})$ low-frequency drivers. The speakers bring the platform into a vibrational-rotational movement, to which they are connected by rods through the frictionless joints (Fig. 7). The key principles of work and the practical basis of calibration are described in Abramovich et al. (1997). The water pipe was fixed on the vibration platform with the possibility of vertical movement. An eight-channel 16-bit data acquisition system, NI USB-6215 (Bus-Powered M Series Multifunction DAQ for USB-16-Bit, 2018), (3) was used to collect the signal from the reference sensors and the

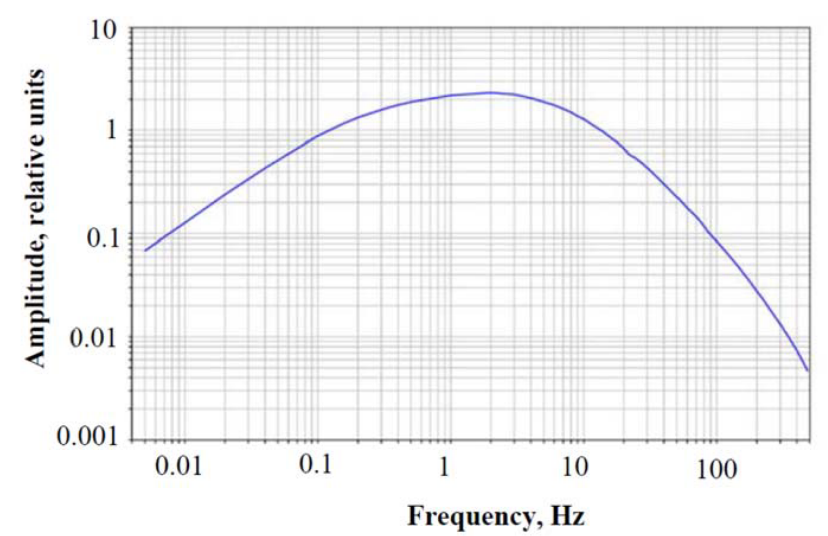

Figure 8. Self-calibration METH $W_{\mathrm{s}} W_{\mathrm{f}} W_{\mathrm{fb}}$ amplitude vs. frequency response. $\mathrm{Hz}$ on the $x$ axis, relative units on the $y$ axis.

output signals of the hydrophones. This was the striate way to make pressure variations with the known amplitude at different frequencies. Vertical water column displacement $X(w)$ was measured with precise displacement sensors of the calibrating platform and the corresponding pressure was calculated according to the ratio $P(\omega)=X(\omega) S_{\text {pipe }}\left(S_{\text {pipe }}\right.$ is the water surface).

There are other ways to calibrate the MET hydrophone, and one of the main aims of the present research was to investigate the simplest way of METH calibration. So, we have compared all the techniques and present the results for later discussion.

The second and prior way for closed-loop METH calibration is self-test. The METH frequency response can be described by the mathematical model (1). The amplitude and the phase-frequency response of the function $W_{\mathrm{s}} W_{\mathrm{f}} W_{\mathrm{fb}}$ were measured to study self-test calibration with feedback board cascade help. $W_{\mathrm{s}}$ signifies the transfer function of METH; $W_{\mathrm{f}}$ and $W_{\mathrm{fb}}$ are the feed-forward and feedback electronic transfer functions. For that, a harmonic signal taken from DAC NI-6218 was directed on the input $W_{\mathrm{fb}}$ (input FB pin in Fig. 4) and was taken on the output $W_{f}$ (output pin in Fig. 4) at open feedback. Function $W_{\mathrm{s}} W_{\mathrm{f}} W_{\mathrm{fb}}$ amplitude vs. frequency response and hodograph have been constructed. The extracted transfer function is shown in Fig. 8 and the hodograph in Fig. 9. For the convenience of the results' interpretation, the cascades of the accompanying electronic circuit with the transfer characteristics $W_{\mathrm{f}}$ and $W_{\mathrm{fb}}$ have been chosen to be frequency independent. In this case, in fact in Fig. 8, the transfer characteristic of the sensor $W_{\mathrm{s}}$ obtained with accuracy to a numerical coefficient is presented. It can be seen that the shape of the characteristic corresponds to the theoretical model in Fig. 5 for $\mathrm{pV}=4$ and $p=1$ atm, which is well correlated with the parameters of the experimental sample.

In accordance with the corollary of the stability criterion for the Nyquist-Mikhailov dynamical system, if the open 


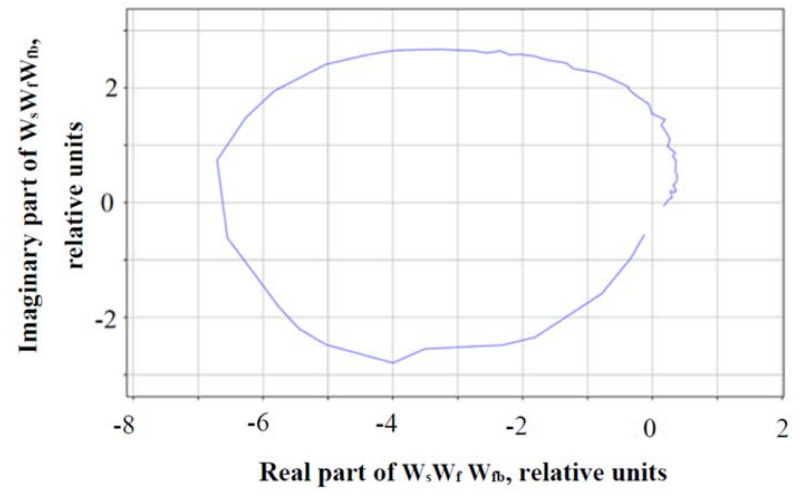

Figure 9. METH $W_{\mathrm{s}} W_{\mathrm{f}} W_{\mathrm{fb}}$ amplitude-phase-frequency response (hodograph) in the complex plane.

system with the transfer function $W(j \omega)$ is stable, the closed system is stable if the hodograph of the open system does not cover the point $(-1 ; j 0)$. Based on this criterion and the data in Fig. 9, the dynamic METH system with closed feedback will be stable.

The third way is to make the strong pressure variations by the vertical water column displacements and find the spectrum ratio of the METH signal and the reference hydrophone signal BC-311 (BC 311 Underwater/threaded hydrophone, 2018); see Fig. 10. The METH and BC-311 were placed close to each other. Using the assembly from Fig. 7, strong shift signals were excited at the resonance frequency of the vibrating table, which were fixed by two closely located hydrophones. The strong signal was registered in the recorded spectrum over a wide frequency band; as can be seen from the analysis of Fig. 10, the red and blue spectra are strongly correlated, practically in the entire frequency band. Assuming a frequency response of the reference hydrophone BC311 flat, the relationship between the signals from the correlated spectra of the studied METH to the spectrum of BC-311 with a known flat transfer function has been found. As a result, the METH transfer characteristic in the blue graph in Fig. 11 has also been obtained.

The results for comparison of self-test METH calibration with the calibration by the calibrating platform and reference hydrophone at low-frequency amplitude response are given in Fig. 11. The green curve is the METH platform calibration, the blue curve is the strong signal calibration with $\mathrm{BC}$ 311 , and the red curve is the self-test calibration calculated in the $\mu \mathrm{V} \mathrm{Pa}^{-1}$ respectively.

Based on the comparison shown in Fig. 11, we can conclude that all the proposed METH calibration methods are equivalent in terms of the result and give the same soughtafter METH frequency response. The technically simplest method of calibration can be used hereafter, which is selfcalibration by the coil.

In accordance with the above results, full correspondence of all types of calibration techniques can be observed. But

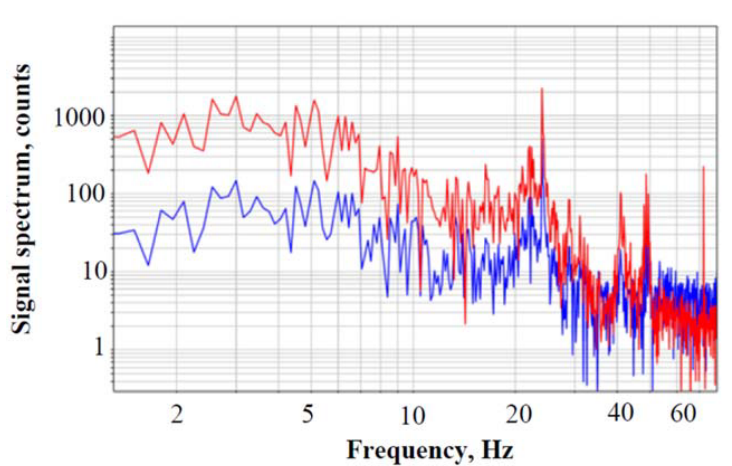

Figure 10. Pressure variations at the $24 \mathrm{~Hz}$ signal. Red is the MET hydrophone signal spectrum, and blue is the ZETLab BC-311 hydrophone signal spectrum. Hz on the $x$ axis, counts of DAS on the $y$ axis.

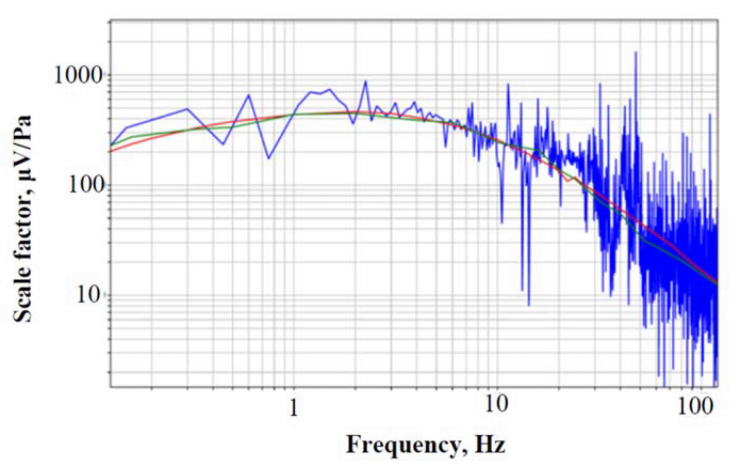

Figure 11. METH self-test calibration (red) with METH calibrating platform calibration (green) and a strong signal calibration curve (blue). $\mathrm{Hz}$ on the $x$ axis, $\mu \mathrm{V} \mathrm{Pa}^{-1}$ on the $y$ axis.

it is more convenient to use sensors with a flat frequency response. To do so, special electronic nominal have been found (according to the scheme in Fig. 4). To make the flat-frequency response from 0.02 to $200 \mathrm{~Hz}$, specific circuit parameters have been chosen. The experimental results of METH sensitivity with closed-loop feedback are shown in Fig. 12. Self-test calibration has been made under water.

This way, the use of absolute values of water column surface $S$, the values of precise displacement sensor sensitivity for the calibration platform, the sensitivity of the reference hydrophone BC-311, and the METH sensitivity have been measured in the frequency range from 0.02 to $200 \mathrm{~Hz}$, and its absolute value is $0.75 \mathrm{mV} \mathrm{Pa}^{-1}$.

Thus, the present study demonstrated the possibility of creating a hydrophone with a high sensitivity in the region of ultra-low acoustic frequencies, and experimentally proved the identity of the calibrations by different schemes. 


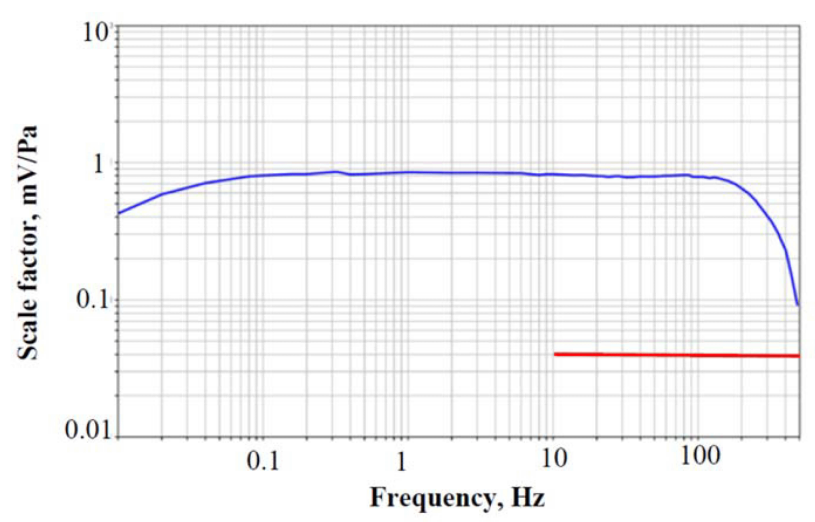

Figure 12. METH closed-loop feedback self-test calibration (blue curve) with BC-311 sensitivity level (red curve). $\mathrm{Hz}$ - on the $x$ axis; $\mathrm{mV} \mathrm{Pa}^{-1}-$ on the $y$ axis.

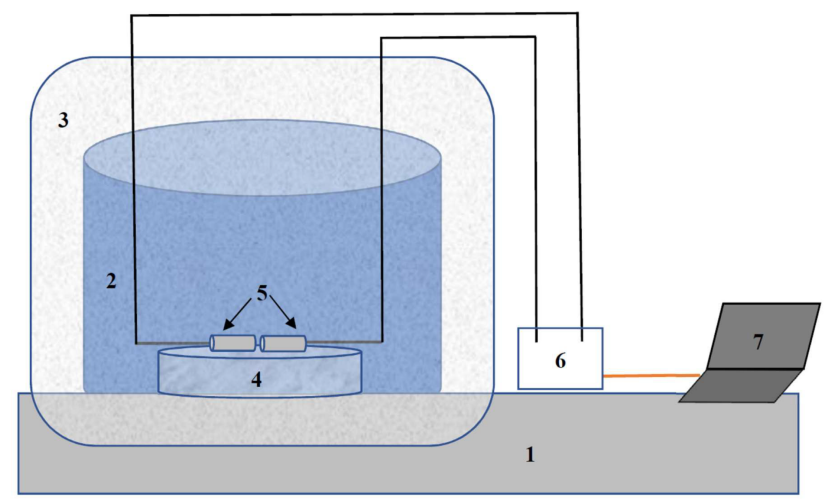

Figure 13. Installation for measuring the intrinsic noise of molecular-electronic hydrophones. 1 - unbundled seismic foundation, 2 - thick-walled metal tank with water, 3 - foam cap, 4 - foam base, 5 tested molecular and electronic hydrophones, 6 - data acquisition system, 7 - laptop with special software.

\subsection{Experimental noise research}

For experimental noise measurements, two METHs with an identical sensitivity of $0.75 \mathrm{mV} \mathrm{Pa}^{-1}$ within a frequency range between 0.02 and $200 \mathrm{~Hz}$ have been taken. METHs were placed in a thick-walled metal container with thick walls full of water and this container was covered with foam for temperature variation decrease, and the signals were recorded for several night hours with the help of the NDAS data acquisition system (24 bit) (NDAS-8224 4-channel seismic signals recorder, 2018); see Fig. 13.

The METH's signal power spectral densities are shown in Fig. 14 . We research only a quiet period of the night's recording. In Fig. 14, the red and blue colours show power spectral densities of the two identical METHs that are placed coaxially and close to each other, while the noise power spectral density of the data acquisition system in units of pressure is green. The violet curve represents the non-correlative part of

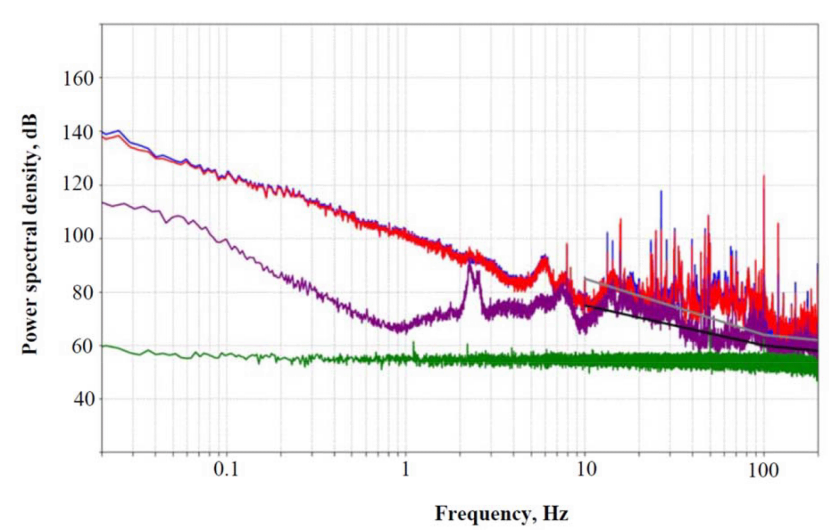

Figure 14. METH night test. Red and blue are the PSD of close METHs, green is the ADC self-noise, purple is the uncorrelated part corresponding to METH self-noise, black is sea state zero, and grey is the TC4035 Teledyne Reson hydrophone. $\mathrm{Hz}-x$ axis, $\mathrm{dB}$ (re $1 \mu \mathrm{Pa} / \sqrt{\mathrm{Hz}})-y$ axis.

the observed METH's night period signals that mean the selfnoise of the studied METH, and it was calculated according to the equation of Egorov et al. (2017):

$$
\begin{aligned}
& \left\langle\left|U_{N}(\omega)\right|^{2}\right\rangle=\frac{\sqrt{\left\langle\left|U_{1}(\omega)\right|^{2}\right\rangle\left\langle\left|U_{2}(\omega)\right|^{2}\right\rangle}}{|W(\omega)|^{2}} \\
& \times\left(1-\sqrt{\frac{\left\langle U_{1}^{*}(\omega) U_{2}(\omega)\right\rangle\left\langle U_{1}(\omega) U_{2}(\omega)\right\rangle}{\left\langle\left|U_{1}(\omega)\right|^{2}\right\rangle}\left\langle\left|U_{2}(\omega)\right|^{2}\right\rangle}\right),
\end{aligned}
$$

where $U_{1}, U_{2}$ denote signals taken from each METH; $U_{1}^{*}$, $U_{2}^{*}$ denote complex conjugate signals taken from each of the METHs. $W$ denotes the METH transfer function.

Actually, there should be two self-noise curves since the self-noises of the two devices were not exactly the same, but according to Egorov et al. (2017) Eq. (14) works only in assumption of an equivalent-level self-noise of two correlated sensors.

The comparison of the theoretical and experimental results shows their close correspondence, despite the high signal level in the frequency range of $1-40 \mathrm{~Hz}$, which may mean an insufficiently quiet place and environment for a noise test. That result limits the top METH noise level and could be reduced in the next studies.

\section{Conclusions}

The main result of the research is the theoretical and experimental model of METH, methods and the technique for low-frequency calibration, and the experimental structure of closed-loop feedback METH with a $0.02-200 \mathrm{~Hz}$ flatfrequency response with a sensitivity level of $0.75 \mathrm{mV} \mathrm{Pa}^{-1}$. The identity of the results of calibrations by different methods has been shown. A mathematical theoretical model describing the behaviour of amplitude-frequency responses as 
a function of system parameters has been confirmed, including the exceptionally high sensitivity of METH. The theoretical METH self-noise floor in the low-frequency range has been shown to be able to be much lower even than the Wenz model. That makes it possible to increase the standard hydrodynamic resistance of the transforming electrode cell by more than 80 times. On the one hand, this will lead to an increase in the hydrodynamic intrinsic noise, to the level of the pressure variations that are minimally recorded in the ocean, and on the other hand to a significant shift in the noise of the convective type to the region of higher frequencies and to their total decrease. In addition, strong damping will give a wide and flat transfer characteristic of the mechanical system, which will allow us to expand the working frequency band in the region of lower and higher frequencies.

Another significant conclusion, according to Eq. (8), represents the mechanical part of the transfer function of the MET hydrophone; the mass of the electrolyte is cancelled out in Eq. (8). The physical mechanisms of noise presented in the theoretical section are not affected by the mass and overall dimensions of the hydrophone. So, it seems possible to significantly reduce the overall dimensions of the MET hydrophone. The level of self-noise and sensitivity of the hydrophone will not be significantly affected by the mass of the electrolyte.

Data availability. The underlying measurement data are not publicly available and can be requested from the authors if required.

Author contributions. DLZ - obtaining experimental data and developing the experimental set-up; SYA - developing the structure scheme of METH; MAR - calculating the theoretical dependence of the METH transfer function and METH self-noise; IE - getting experimental data; EVE and VMA - making a sufficient contribution in discussing the results and conclusion.

Competing interests. The authors declare that they have no conflict of interest.

Acknowledgements. This work was supported by the Russian Ministry of Education and Science state assignment under grant 3.3197.2017.

Edited by: Andreas Schütze

Reviewed by: two anonymous referees

\section{References}

Abramovich, I., Agafonov, V., Daragan, S., and Kazak, B.: Wide Band Motion Sensor Calibrator, Seismol. Res. Lett., 67, 34-38, 1997.

Abramovich, I. A. and Kharlamov, A. V.: Electrochemical transducers and a method for fabricating the same, U.S. Patent 6576103 B2, 2003.

Agafonov, V., Antonov, A., Razin, A., Avdyukhina, S., Egorov, I., Neeshpapa, A., and Dmitry, P.: Low-frequency sea-bottom seismic station for offshore exploration, 9th Congress of the Balkan Geophysical Society, BGS-2017, November, 2017.

Agafonov, V. M., Egorov, I. V., and Shabalina, A. S.: Operating principles and specifications of small-size molecular electronic seismic sensor with negative feedback, Seismic Instrum., 49, 519, 2013.

Antonovskaya, G. N., Kapustian, N. K., Moshkunov, A. I., Danilov, A. V., and Moshkunov, K. A.: New seismic array solution for earthquake observations and hydropower plant health monitoring, J. Seismol., 21, 1039, https://doi.org/10.1007/s10950-0179650-8, 2017.

Asolkar, P., Das, A., Gajre, S., and Joshi, Y.: Comprehensive correlation of ocean ambient noise with sea surface parameters, Ocean Eng., 138, 170-178, 2017.

BC 311 Underwater/threaded hydrophone: BC-311 datasheet, Zet Lab publication, available at: https://zetlab.com/en/shop/sensors/ bc-311-underwater-threaded-hydrophone/, last access: 12 July 2018.

Bradley, D. L. and Nichols, S. M.: Worldwide Low-Frequency Ambient Noise, Acoustics Today, 11, 20-26, 2015.

Bus-Powered M Series Multifunction DAQ for USB-16-Bit: NI USB-6215 datasheet, National Instruments publication, available at: http://www.ni.com/datasheet/pdf/en/ds-9, last access: 12 July 2018.

de Medeiros, L. J. , Kamimura, H. A. S., Altafim, R. A. P., Carneiro, A. A. O., Amorim, M. F., and Altafim, R. A. C.: Piezoelectret-based hydrophone: an alternative device for vibro-acoustography, Meas. Sci. Technol, 26, 095102, https://doi.org/10.1088/0957-0233/26/9/095102, 2015.

Deng, T., Chen, D., Chen, J., Sun, Z., and Wang, J.: Microelectromechanical Systems-Based Electrochemical Seismic Sensors With Insulating Spacers Integrated Electrodes for Planetary Exploration, IEEE Sens. J., 16, https://doi.org/10.1109/JSEN.2015.2491783, 2016.

Egorov, E. V., Kozlov, V. A., and Yashkin, A. V.: Phase-Response Characteristics of the Transfer Function of a Spatially Limited Electrochemical Cell, Russ. J. Electrochem., 43, 1358-1363, https://doi.org/10.1134/S102319350712004X, 2007.

Egorov, I. V., Shabalina, A. S., and Agafonov, V. M.: Design and Self-Noise of MET Closed-Loop Seismic Accelerometers, IEEE Sens. J., 17, 7, https://doi.org/10.1109/JSEN.2017.2662207, 2017.

Gautschi, G.: Piezoelectric Sensorics, Springer-Verlag, Berlin, Heidelberg, 2002.

Grasland-Mongrain, P., Mari, J.-M., Gilles, B., Chapelon, J.-Y., and Lafon, C.: Electromagnetic hydrophone with tomographic system for absolute velocity field mapping, Appl. Phys. Lett., 100, 243502, https://doi.org/10.1063/1.4726178, 2012. 
Huang, H., Liang, M., Tang, R. J. O., and Yu, H.: Molecular Electronic Transducer-Based Low-Frequency Accelerometer Fabricated With Post-CMOS Compatible Process Using Droplet as Sensing Body, IEEE Electr. Device L., 34, 1304-1306, 2013.

Hurd R. M. and Lane, R. N.: Principles of Very Low Power Electrochemical Control Devices, J. Electrochem. Soc., 104, 727-730, 1957

Kim, K. S., Mizuno, Y., and Nakamura, K.: Fiber-optic ultrasonic hydrophone using short Fabry-Perot cavity with multilayer reflectors deposited on small stub, Ultrasonics, 54, 1047-1051, 2014.

Kopp, L., Cano, D., Dubois, E., Wang, L., Smith, B., and Coates, R. F. W.: Potential performance of parametric communications, IEEE J. Oceanic Eng., 25, 282-295, 2000.

Kozlov, V. A. and Sakharov, K. A.: Self-noise of molecularelectronic transducers of diffusion type, The physical basis for liquid and solid measurement systems and devices of information processing, MIPT, 43-49, 1994.

Kozlov, V. A. and Safonov, M. V.: Self-noise of molecular electronic transducers, Experim. Instrum. Techn., 73, 81-84, 2003.

Kozlov, V. A. and Terent'ev, D. A.: Transfer function of a diffusion transducer at frequencies exceeding the thermodynamic frequency, Russian J. Electrochem., 39, 401-406, 2003.

Larcam, C. W.: Theoretical analysis of the solution solion polarised cathode acoustic linear transduser, J. Acoust. Soc. Am., 37, 664678, 1965.

Lasky, M., Richard Doolittle, D., Simmons, B. D., and Lemon, S. G.: Recent progress in towed hydrophone array research, IEEE J. Oceanic Eng., 29, 374-387, 2004.

Lidorenko, N. S., Ilin, B. I., Zaidenman, I. A., Sobol, V. V., and Shchigorev, I. G.: An Introduction to Molecular Electronics, Moscow, Russia, Energoatomizdat, 320 pp., 1984.

Liu, D., Liang, Y., Jin, L., Sun, H., Cheng, L., and Guan, B.-O.: Highly sensitive fiber laser ultrasound hydrophones for sensing and imaging applications, Opt. Lett., 41, https://doi.org/ 10.1364/OL.41.004530, 2016.

Ma, J., Zhao, M., Huang, X., Chen, H. B. Y., and Yu, M.: Low cost, high performance white-light fiberoptic hydrophone system with a trackable working point, Opt. Exp., 24, https://doi.org/10.1364/OE.24.019008 2016.

Mismund, O. A.: Underwater acoustics in marine fisheries and fisheries research, Rev. Fish Biol. Fish., 7, 1-34, 1997.

NDAS-8224 4-channel seismic signals recorder: NDAS- 8224 datasheet, R-sensors publication, available at: http://r-sensors. ru/1_products/Descriptions/NDAS-8224.pdf, last access: 12 July 2018.

Safonov, M. V.: Noises in the electrochemical cell caused by free convection of the liquid in the gravity field, Proceedings of the XLVI scientific conference MIPT "Modern problems of fundamental and applied sciences", Part V, Quantum and physical electronics, Moscow, Dolgoprudny, 2003.
Shabalina, A. S.: Methods for improving noise characteristics at high frequencies in molecular-electronic geophones, Proceedings of the XLIX scientific conference MIPT "Modern problems of fundamental and applied sciences", Part V, Quantum and physical electronics, Moscow, Dolgoprudny, 2007.

Shen, B., Wada, Y., Koyama, D., Isago, R., Mizuno, Y., and Nakamura, K.: Fiber-optic ultrasonic probe based on refractive-index modulation in water, in Proceedings of SPIE7753 21st International Conference on Optical Fiber Sensors, 7753, 77539W, 2011.

Sherman, C. H. and Butler, J. L.: Transducers and Arrays for Underwater Sound, Springer, New York, 2007.

Slotte, A., Hansen, K., Dalen, J., and Ona, E.: Acoustic mapping of pelagic fish distribution and abundance in relation to a seismic shooting area off the Norwegian west coast, Fish. Res., 67, 3150, https://doi.org/10.1016/j.fishres.2003.09.046, 2004.

Tan, Y. , Zhang, Y., and Guan, B.: Hydrostatic pressure insensitive dual polarization fiber grating laser hydrophone, IEEE Sens. J., 11, 1169-1172, 2011.

Teledyne Marine publication: TC-4032 datasheet, available at: http: //www.teledynemarine.com/reson-tc-4032?ProductLineID=48, last access: 12 July 2018.

Xu, W., Liu, Y., Zhang, G., Wang, R., Xue, C., Zhang, W., and Liu, J.: Development of cup-shaped micro-electromechanical systems-based vector Hydrophone, J. Appl. Phys., 120, 124502, https://doi.org/10.1063/1.4962859, 2016.

Wenz, G. M.: Acoustic Ambient Noise in the Ocean: Spectra and Sources, J. Acoust. Soc. Am., 34, 1936-19556, 1962.

Zaitsev, D., Agafonov, V., Egorov, E., Antonov, A., and Shabalina, A.: Molecular electronic angular motion transducer broad band self-noise, Sensors, 15, 29378-29392, 2015.

Zaitsev, D. L., Agafonov, V. M., Egorov, E. V., Antonov, A. N., and Krishtop, V. G.: Precession azimuth sensing with lownoise molecular electronics angular sensors, J. Sensors, 2016, 6148019, https://doi.org/10.1155/2016/6148019, 2016.

Zaitsev, D., Agafonov, V., Egorov, E., and Avdyukhina, S.: Broadband MET Hydrophone 80th EAGE Conference and Exhibition 11-14 June 2018, Copenhagen, Denmark, https://doi.org/10.3997/2214-4609.201801629, 2018a.

Zaitsev, D. L., Agafonov Vadim, M., and Evseev Iliya, A.: Study of Systems Error Compensation Methods Based on MolecularElectronic Transducers of Motion Parameters, J. Sensors, 2018, 6261384, https://doi.org/10.1155/2018/6261384, 2018b. 